\title{
Inhalation of $\alpha-g a l /$ sialic acid liposomes: a novel approach for inhibition of influenza virus infection?
}

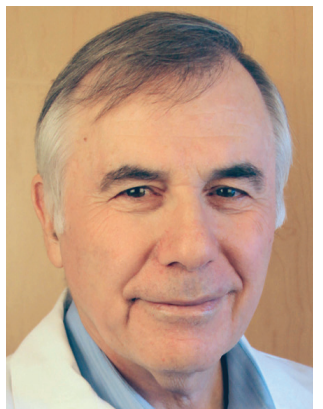

Uri Galili*

\author{
“...inhibition of influenza virus infection in \\ symptomatic patients may be feasible by \\ inhalation of aerosol-containing liposomes \\ presenting $\alpha$-gal epitopes and sialic acid \\ epitopes."
}

First draft submitted: 2 December 2015; Accepted for publication: 8 December 2015; Published online: 3 February 2016

Effective inhibition of influenza virus infection in symptomatic patients may be feasible by inhalation of aerosol containing liposomes presenting $\alpha$-gal epitopes and sialic acid epitopes. The virus binds to sialic acid epitopes and the natural antiGal antibody binds to $\alpha$-gal epitopes on the liposomes and activates the complement system to generate chemotactic peptides that recruit macrophages. These macrophages bind and internalize via their $\mathrm{Fc}$ receptors, anti-Gal-coated liposomes and the influenza virus bound to them, process the viral antigens and transport them to the regional lymph nodes for eliciting a rapid, protective immune response that prevents progression of the virus infection. Treatments for seasonal outbreaks of influenza virus infections are only partially successful. Yearly influenza vaccinations have suboptimal efficacy and $25-50 \%$ of immunized individuals (in particular elderly populations) contract the disease during the influenza season [1]. Neuraminidase inhibitors aimed to slow virus growth at early stages of infection are considered by some reports to be suboptimal as well $[2,3]$. Here, a novel type of therapy for symptomatic flu patients is proposed. It consists of inhalation of aerosolized liposomes called ' $\alpha$-gal/sialic acid liposomes.' This therapy aims to slow progression of influenza virus infection at early stages of the disease and accelerate the induction of protective immune response against the virus, thereby decreasing recovery time, complications, morbidity and mortality.

$\alpha-\mathrm{Gal} /$ sialic acid liposomes are biodegradable liposomes presenting multiple carbohydrate epitopes of two types: $\alpha$-gal epitopes with the structure galactose linked $\alpha 1-3$ to a penultimate galactose on the carbohydrate chain of a glycolipid (e.g., Galo1-3Galß1-4GlcNAc-R) and sialic acid (SA) epitopes in which SA is linked $\alpha 2-6$ or $\alpha 2-3$ to the penultimate galactose on the carbohydrate chain

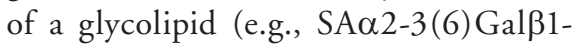
$4 \mathrm{GlcNAc}-\mathrm{R}$ ) (Figure 1). The proposed $\alpha$-Gal/SA liposomes therapy is based on

\section{KEYWORDS}

- $\alpha$-gal epitope - anti-Gal antibody

- infection inhibition • influenza virus

- liposome inhalation

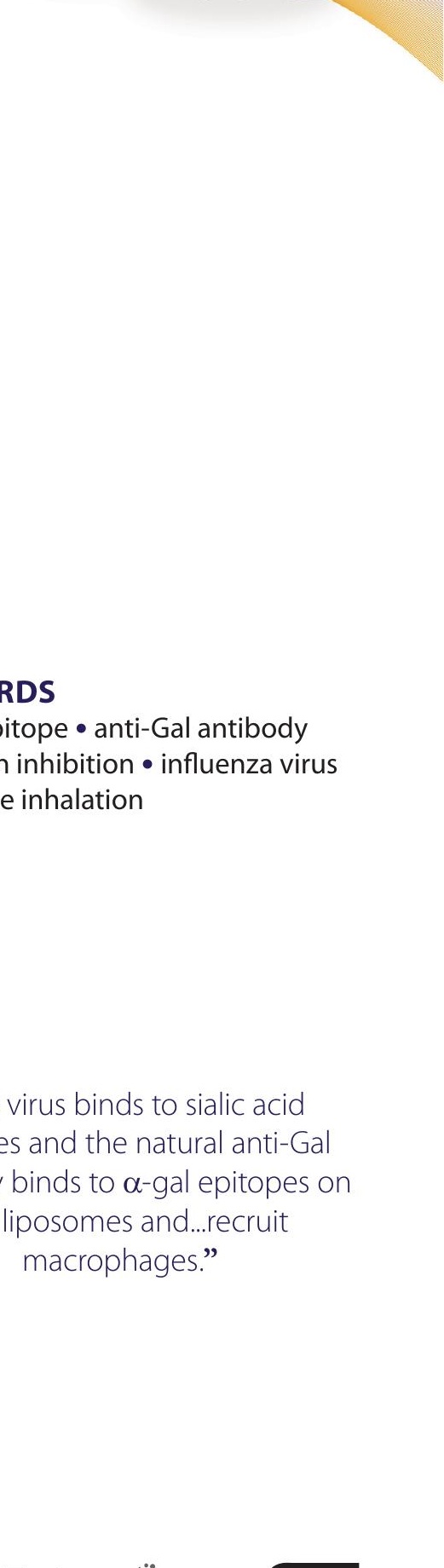


“These macrophages... internalize...anti-Gal-coated liposomes and the influenza virus bound to them, process the viral antigens and transport them to the regional lymph nodes for eliciting a rapid, protective immune response that prevents progression of the virus infection."

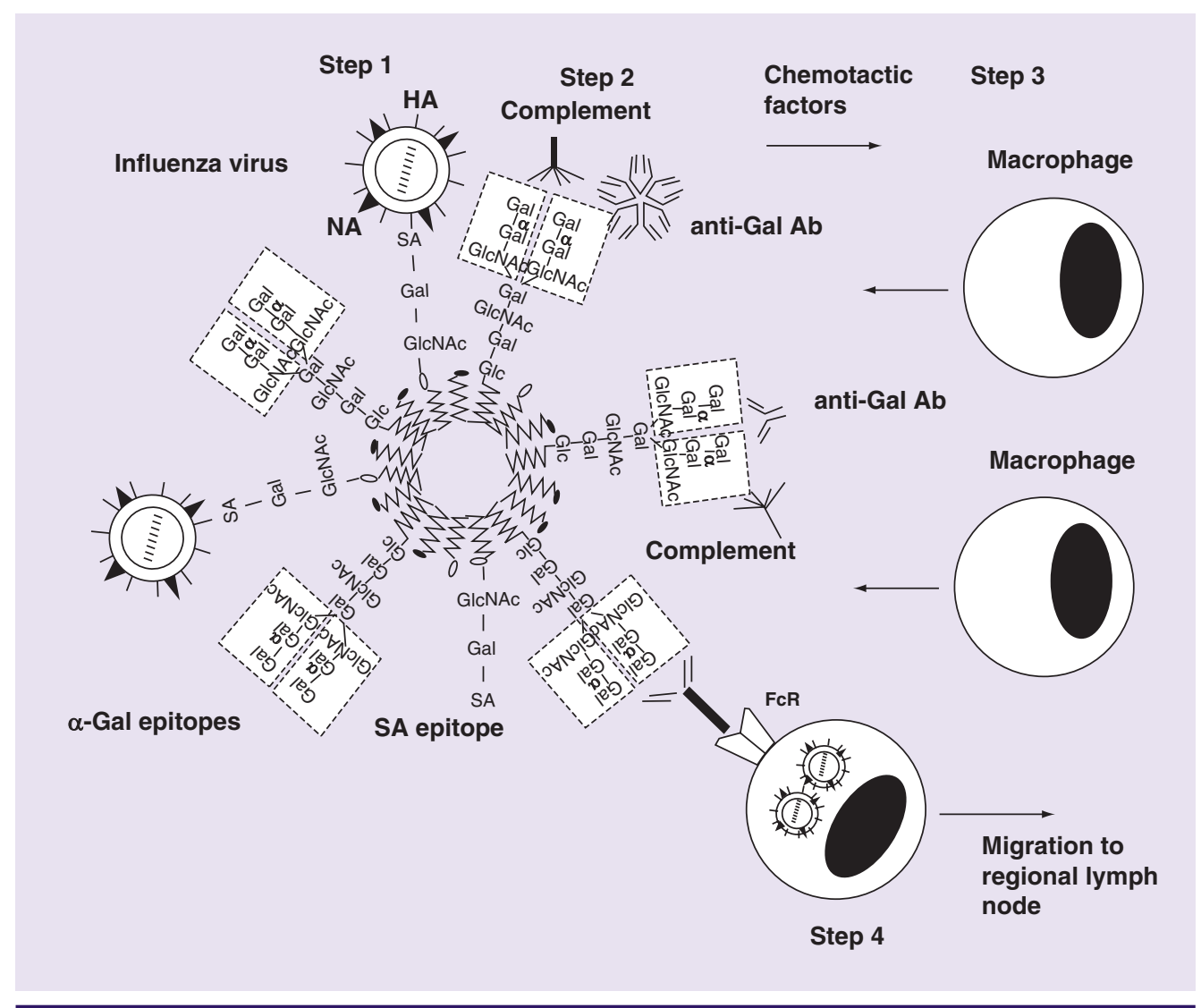

Figure 1. Proposed sequential steps occurring after the inhaled $\alpha$-gal/SA liposomes land in the mucus and surfactant lining of the respiratory tract. The virus binds to SA epitopes on $\alpha-g a l / S A$ liposomes. The natural anti-Gal antibody binds to $\alpha$-gal epitopes on $\alpha$-gal/SA liposomes and activates the complement system to produce chemotactic complement cleavage peptides, such as C5a and C3a. Macrophages are recruited by the chemotactic peptides toward the $\alpha-g a l / S A$ liposomes. Influenza virus bound to $\alpha$-gal/SA liposomes is internalized by macrophages following interaction between the immunocomplexed anti-Gal antibody and FcRs on macrophages. These macrophages function as antigen presenting cells that process the influenza virus antigens and transport them to regional lymph nodes. HA is illustrated as capable of binding SA epitopes. The virus is schematically described with eight pieces of segmented negative sense RNA illustrated as small bars within the virus.

FCR: Fc receptor; HA: Influenza virus hemagglutinin; NA: Influenza virus neuraminidase; SA: Sialic acid.

two phenomena: 1. SA epitopes on cell membrane glycoproteins and glycolipids serve as 'docking receptors' for binding of influenza virus to cells via its hemagglutinin $[4,5]$. 2. $\alpha-\mathrm{Gal}$ epitopes are the ligand for the natural anti-Gal antibody, the most abundant natural antibody in humans, constituting approximately $1 \%$ of serum immunoglobulins [6-8]. Anti-Gal is also present in various human secretions as IgA and $\mathrm{IgG}$ isotypes [9]. $\alpha$-Gal epitopes are naturally expressed in large numbers on cells of nonprimate mammals, prosimians and New World monkeys, but not in Old World monkeys, apes and humans, all of which produce anti-Gal as a natural antibody [10-12]. $\alpha-G a l / S A$ liposomes are produced by drying a mixture of glycolipids carrying $\alpha$-gal epitopes with glycolipids carrying SA epitopes and with phospholipids. This dried mixture is sonicated extensively in saline to produce $\alpha$-gal/SA liposomes.

Infection of respiratory epithelium cells with influenza virus requires binding of the virus to SA epitopes on cells $[4,5]$. Furthermore, such interaction may be inhibited by natural and synthetic soluble molecules expressing multiple SA epitopes [13-16]. Therefore, one may assume that inhalation of liposomes presenting multiple SA epitopes, but lacking $\alpha$-gal epitopes, will 
result in binding of the virus to SA epitopes on the inhaled liposomes, which land in mucus and surfactant films lining the respiratory tract. However, since there is no mechanism to remove the virus bound to such liposomes, the virus may ultimately infect the respiratory epithelium. Presentation of both $\alpha$-gal and SA epitopes on liposomes (i.e., $\alpha$-gal/SA liposomes) may enable harnessing the natural anti-Gal antibody for destruction of influenza virus bound to such liposomes and may accelerate the protective immune response against the virus.

In the proposed therapy, $\alpha$-gal/SA liposomes are administered by inhalation of aerosol to patients at early stages of influenza virus infection. The inhaled $\alpha$-gal/SA liposomes are trapped in mucus film lining the respiratory epithelium and in the surfactant lining the alveoli. These liposomes slow or prevent progression of the virus infection by the following sequential steps (Figure 1):

1. Influenza virus within the mucus and surfactant lining the respiratory tract binds to the multiple SA epitopes on $\alpha$-gal/SA liposomes, thus the virus may be prevented from further infection of cells.

2. Natural anti-Gal IgG and IgA antibodies released into the infected lungs bind to the multiple $\alpha$-gal epitopes on $\alpha$-gal/SA liposomes and activate the complement system to produce chemotactic complement cleavage peptides, such as $\mathrm{C} 5 \mathrm{a}$ and $\mathrm{C} 3 \mathrm{a}$. No cells are damaged by this complement activation since it occurs on the surface of liposomes.

3. Macrophages are recruited by these chemotactic peptides and migrate toward the inhaled $\alpha$-gal/SA liposomes.

4. $\alpha-G a l / S A$ liposomes with bound influenza virus are internalized by recruited macrophages as a result of interaction between immunocomplexed anti-Gal antibodies on liposomes and Fc- $\gamma$ receptors or Fc- $\alpha$ receptors on macrophages. Internalized influenza virus is killed within these macrophages, which further function as antigen-presenting cells (APC), processing virus peptides and transporting them to regional lymph nodes.

5. The macrophages present processed virus peptides to $T$ cells within the regional lymph nodes and elicit protective humoral and cellular immune responses (Step 5 is not included in Figure 1).

Both binding of influenza virus to inhaled $\alpha$-gal/SA liposomes and anti-Gal-mediated effective uptake of liposomes bound virus by macrophages decrease virus burden in the respiratory tract and thus decrease damage to the respiratory tract epithelium, as well as slows the progression of the infection. Moreover, the rapid and effective processing and transport of virus immunogenic peptides to regional lymph nodes induce an accelerated, effective immune response that may stop progression of the virus infection faster than the physiologic immune response, thereby decreasing the disease period. This accelerated immune response includes extensive activation of helper $\mathrm{T}$ cells, which help B cells to produce anti-influenza virus IgA antibodies capable of neutralizing the virus in the respiratory tract. In addition, macrophages presenting influenza virus peptides activate virus-specific cytotoxic $T$ cells (CTL) that proliferate in lymph nodes, then circulate and kill virus infected cells. By killing infected cells, these CTL prevent further expansion of the viral infection in healthy cells.

The efficacy of inhaled $\alpha$-gal/SA liposomes in slowing progression of influenza virus infection and shortening the infection time will be evaluated in future studies. However, several observations support the hypothesis presented in Figure 1. Step 1 in that figure predicts influenza virus binding to SA epitopes on $\alpha$-gal/SA liposomes. This can be inferred from the wellestablished function of SA epitopes as receptors for the virus on cells $[4,5]$ and from the ability of natural and synthetic SA epitopes to inhibit binding of this virus to its receptors [13-16].

Steps 2 and 3 predicting interaction of antiGal with $\alpha$-gal epitopes on $\alpha$-gal/SA liposomes and the resulting recruitment of macrophages are supported by observations on the interaction of this antibody with liposomes presenting multiple $\alpha$-gal epitopes ( $\alpha$-gal liposomes) in studies in $\alpha 1$,3galactosyltransferase knockout mice (GT-KO) mice. These mice produce anti-Gal in titers comparable to those in humans [17,18]. Cutaneous administration of $\alpha$-gal liposomes into GT-KO mice results in binding of anti-Gal to these liposomes, followed by activation of the complement system and generation of complement chemotactic cleavage peptides that induce rapid recruitment of macrophages $[17,18]$. “...the studies on ovalbumin encapsulated in $\alpha$-gal liposomes...and on vaccinating influenza virus engineered to express $\alpha$-gal epitopes...strongly suggest that anti-Galmediated targeting to macrophages of infective influenza virus bound to $\alpha$-gal/SA liposomes will result in effective $\mathrm{Fc} / \mathrm{FC}$ receptor uptake of the virus by macrophages." 
Step 4 predicts internalization of $\alpha$-gal/SA liposomes and bound influenza virus, after interaction between the Fc portion of anti-Gal on these liposomes and Fc receptors on macrophages. This binding and internalization were demonstrated with $\alpha$-gal liposomes encapsulating ovalbumin (OVA) and coated with the antiGal antibody. When coincubated with GT-KO mouse macrophages, such liposomes readily bound to the macrophages and internalized by them [19]. The study with $\alpha$-gal liposomes encapsulating OVA further demonstrated in vitro and in vivo a much more effective transport, processing and presentation of immunogenic OVA peptides when the encapsulated OVA within the $\alpha$-gal liposomes was targeted by anti-Gal for uptake by APC, such as dendritic cells and macrophages [19]. Vaccination with OVA encapsulated within $\alpha$-gal liposomes resulted in approximately 30 -fold higher anti-OVA immune response in mice producing anti-Gal than in mice lacking this targeting antibody. This study further supports the prediction of Step 5 above, which suggests that anti-Gal-mediated targeting of influenza virus bound to $\alpha$-gal/SA liposomes for uptake by macrophages, may increase the immunogenicity of the virus peptides. This increase is the result of effective transport to lymph nodes, processing and presentation of these peptides by the recruited macrophages [19].

The prediction in Step 5 is also supported by studies on vaccination of anti-Gal producing GT-KO mice immunized with inactivated A/Puerto Rico/8/34-H1N1 (PR8) influenza virus engineered to express $\alpha$-gal epitopes. Vaccinating virus expressing $\alpha$-gal epitopes induced anti-PR8 virus immune response 30 to 100 -fold higher than that in mice immunized with parental influenza virus lacking $\alpha$-gal epitopes [20]. The improved anti-Gal-mediated uptake by APC, processing and presentation of PR8 virus expressing $\alpha$-gal epitopes was demonstrated in in vitro studies as well [21]. Accordingly, intranasal challenge with live PR8 virus resulted in survival of approximately $90 \%$ of mice immunized with inactivated viruspresenting $\alpha$-gal epitopes, whereas only $10 \%$ of mice immunized with PR8 virus lacking $\alpha$-gal epitopes survived the challenge [20].

Overall, the studies on OVA encapsulated in $\alpha$-gal liposomes [19] and on vaccinating influenza virus engineered to express $\alpha$-gal epitopes [20] strongly suggest that anti-Galmediated targeting to macrophages of infective influenza virus bound to $\alpha$-gal/SA liposomes will result in effective $\mathrm{Fc} / \mathrm{F}_{\mathrm{c}}$ receptor uptake of the virus by macrophages. This uptake will be followed by effective transport, processing and presentation of the immunogenic peptides for the activation of virus-specific $T$ cells within the regional lymph nodes, ultimately eliciting an anti-influenza virus protective immune response that decreases the disease period, morbidity and complications caused by influenza virus infection. Since some influenza virus strains bind preferentially to terminal $S A \alpha 2-3$ residues and other to $S A \alpha 2-6$ residues of the SA epitope [13-16,22], it is further suggested that $\alpha$-gal/SA liposomes may be optimized for multiple strains by presenting both $S A \alpha 2-3$ and SA $2-6$ terminal residues.

\section{Financial \& competing interests disclosure}

$U$ Galili has submitted a provisional patent application (application number 62/177,115) to the USA Patent Office, which is currently pending. The author has no other relevant affiliations or financial involvement with any organization or entity with a financial interest in or financial conflict with the subject matter or materials discussed in the manuscript apart from those disclosed.

No writing assistance was utilized in the production of this manuscript.

\section{References}

1 Webster RG. Immunity to influenza in the elderly. Vaccine 18, 1686-1689 (2000).

2 Coenen B, Van Puyenbroeck K, Verhoeven V et al. The value of neuraminidase inhibitors for the prevention and treatment of seasonal influenza: a systematic review of systematic reviews. PLoS ONE 8(4), e60348 (2013).

3 Okoli GN, Otete HE, Beck CR, Nguyen-VanTam JS, Schmidt RL. Use of neuraminidase inhibitors for rapid containment of influenza: a systematic review and meta-analysis of individual and household transmission studies. PLoS ONE 9(12), e113633 (2014).

4 Johnson CA, Pekas DJ, Winzler RJ. Neuraminidase and influenza virus infection in embryonated eggs. Science 143, 1051-1052 (1964).

5 Skehel JJ, Wiley DC. Receptor binding and membrane fusion in virus entry: the influenza hemagglutinin. Annu. Rev. Biochem. 69, 531-569 (2000).

6 Galili U. Macher BA, Buehler JS, Shohet B. Human natural anti- $\alpha$-galactosyl IgG. II. The specific recognition of $\alpha(1-3)$-linked galactose residues. J. Exp. Med. 162, 573-582 (1985).

7 Galili U, Rachmilewitz EA, Peleg A, Flechner I. A unique natural human IgG antibody with anti- $\alpha$-galactosyl specificity. J. Exp. Med. 160, 1519-1531 (1984).

8 Galili U. Anti-Gal: an abundant human natural antibody of multiple pathogeneses and clinical benefits. Immunology 140, 1-11 (2013).

9 Hamadeh RM, Galili U, Zhou P, Griffis JM. Human secretions contain IgA, IgG and IgM anti-Gal (anti- $\alpha$-galactosyl) antibodies. 
Clin. Diagnos. Lab. Immunol. 2, 125-131 (1995).

10 Galili U, Clark MR, Shohet SB, Buehler J, Macher BA. Evolutionary relationship between the anti-Gal antibody and the Gala1-3Gal epitope in primates. Proc. Natl Acad. Sci. USA 84, 1369-1373 (1987).

11 Galili U, Shohet SB, Kobrin E, Stults CLM, Macher BA. Man, apes, and Old World monkeys differ from other mammals in the expression of $\alpha$-galactosyl epitopes on nucleated cells. J. Biol. Chem. 263, 17755-17762 (1988).

12 Teranishi K, Manez R, Awwad M, Cooper DK. Anti-Gal $\alpha 1-3$ Gal IgM and IgG antibody levels in sera of humans and old world non-human primates. Xenotransplantation 9, 148-154 (2002).

13 Unverzagt C, Kelm S, Paulson JC. Chemical and enzymatic synthesis of multivalent sialoglycopeptides. Carbohydr. Res. 251, 285-301 (1994).
14 Mochalova L, Gambaryan A, Romanova J et al. Receptor-binding properties of modern human influenza viruses primarily isolated in Vero and MDCK cells and chicken embryonated eggs. Virology 313, 473-480 (2003).

15 Matrosovich M, Klenk HD. Natural and synthetic sialic acid-containing inhibitors of infuenza virus receptor binding. Rev. Med. Virol. 13, 85-97 (2003).

16 Olofsson S, Bergström T. Glycoconjugate glycans as viral receptors. Ann. Med. 37, 154-172 (2005).

17 Galili U, Wigglesworth K, Abdel-Motal UM. Accelerated healing of skin burns by anti-Gal/ $\alpha$-gal liposomes interaction. Burns 36, 239-251 (2010).

18 Wigglesworth KM, Racki WJ, Mishra R, Szomolanyi-Tsuda E, Greiner DL, Galili U. Rapid recruitment and activation of macrophages by anti-Gal/ $\alpha$-Gal liposome interaction accelerates wound healing. J. Immunol. 186, 4422-4432 (2011).
19 Abdel-Motal UM, Wigglesworth K, Galili U. Mechanism for increased immunogenicity of vaccines that form in vivo immune complexes with the natural anti-Gal antibody. Vaccine 27, 3072-3082 (2009).

20 Abdel-Motal UM, Guay HM, Wigglesworth K, Welsh RM, Galili U. Increased immunogenicity of influenza virus vaccine by anti-Gal mediated targeting to antigen presenting cells. J. Virol. 81, 9131-9141 (2007).

21 Galili U, Repik PK, Anaraki F, Mozdzanowska K, Washko G, Gerhard W. Enhancement of antigen presentation of influenza virus hemagglutinin by the natural anti-Gal antibody. Vaccine 14, 321-328 (1996).

22 Rogers GN, Paulson JC. Receptor determinants of human and animal influenza virus isolates: differences in receptor specificity of the $\mathrm{H} 3$ hemagglutinin based on species of origin. Virology 127, 361-373 (1983). 